\title{
A Case of Diffuse Large B Cell Lymphoma of the Breast with Predominantly High-Level Internal Echoes
}

\author{
Masafumi Tomita ${ }^{a}$ Shoji Oura ${ }^{b}$ Haruka Nishiguchic \\ Shinichiro Makimoto ${ }^{a}$ \\ aDepartment of Surgery, Kishiwada Tokushukai Hospital, Kishiwada-city, Japan; \\ ${ }^{b}$ Division of Breast Surgery, Kishiwada Tokushukai Hospital, Kishiwada-city, Japan; \\ 'Breast Surgery, Wakaura Chuo Hospital, Wakayama-city, Japan
}

\section{Keywords}

Breast neoplasm · Malignant lymphoma $\cdot$ High-level internal echoes

\begin{abstract}
A 70-year-old woman had a large mass in her right breast. Mammography displayed focal asymmetrical density in the scattered areas of fibroglandular density. Ultrasonography showed the tumor to have predominantly high-level internal echoes. Histological examination showed that the tumor was composed of CD20-positive atypical cells with a large nucleus, scant cytoplasm, and abundant mitoses accompanied by a lot of fat cell interspersion and the diagnosis of diffuse large B cell lymphoma was made. We considered that the massive back scattering generated by the heterogeneity of acoustic impedance between fat cells and tumor cells brought about the high-level internal echoes. The patient had undergone chemotherapy followed by radiotherapy to the breast and regional nodes and has been well without lymphoma recurrence for more than 6 years. Although breast malignant lymphoma generally shows very low-level internal echoes, it could have high-level internal echoes especially in case of a non-dense breast.

\section{Introduction}

Due to its objective nature, mammography is a worldwide mainstay both in the screening $[1,2]$ and clinical examination of the breast. Mammography, however, cannot detect even a large tumor in a so-called dense breast [3] because mammary gland and breast tumor have similar X-ray attenuation coefficients. 
Fig. 1. Mammography showed focal asymmetrical density (arrows) in the right breast with scattered fibroglandular density.

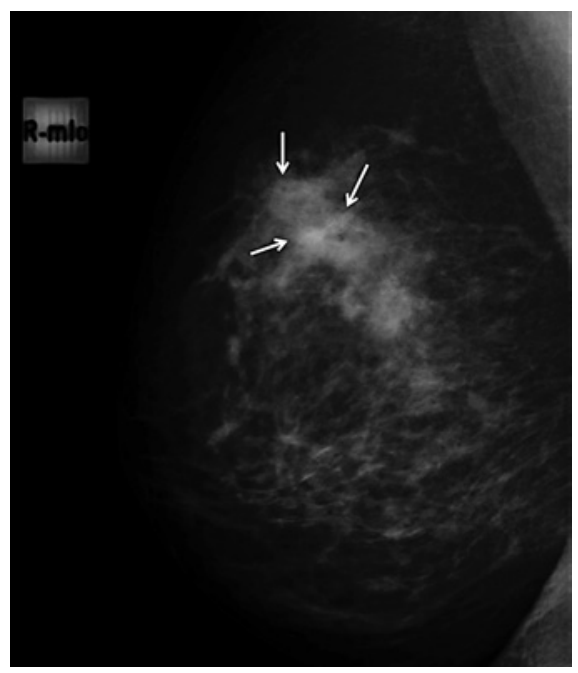

On the other hand, ultrasonography lacks objectivity but can show tomographic images, leading to easy tumor detection even in the dense breast [4]. On making ultrasound diagnosis, physicians routinely pay attention to the shape, margin, internal echoes, depth-width ratio, rupture of the anterior line of the mammary gland, presence of haloes, and posterior echo pattern of the target lesion(s).

Except for apocrine carcinoma, each subtype of breast tumor has typical mammographic and ultrasound images. For example, invasive ductal carcinoma of the scirrhous type [5] generally shows a spiculated mass in mammography and a high depth-width ratio mass with low-level internal echoes and attenuated posterior echoes in ultrasonography.

Breast lymphoma, an extremely rare disease of breast tumors, also has typical image findings such as an expansive growth pattern, a relatively clear margin, enhanced posterior echoes, and very low-level internal echoes [6]. We herein describe a case of a diffuse large B cell breast lymphoma with predominantly high-level internal echoes.

\section{Case Report}

A 70-year-old woman complaining of a right breast mass was referred to our hospital. Mammography, breast composition of Breast Imaging Reporting and Data System category 2 (Fig. 1), showed focal asymmetrical density in the right upper and inner quadrant. Ultrasonography showed a well-demarcated and hypervascular tumor with predominantly highlevel internal echoes (Fig. 2). Magnetic resonance imaging showed a heterogeneously enhanced and well-defined mass. We, therefore, performed vacuum-assisted biopsy to the tumor. Routine histological examination of the tumor revealed atypical cells with a large nucleus, scant cytoplasm, and abundant mitoses accompanied by a lot of fat cell interspersion (Fig. 3a, b). Immunohistochemical examination showed the atypical cells to be AE1 + 3, CAM5.2, CD3, CD5, CD10, CD30, CD56 negative, CD79a weak positive, CD20 positive, and MIB-1 highly positive, leading to the diagnosis of diffuse large B cell lymphoma of the breast. Cytological examination showed no lymphoma infiltration into the bone marrow. Positron emission tomography revealed no distant spread of the breast lymphoma. Under the diagnosis of stage $1 \mathrm{~A}$ diffuse large $\mathrm{B}$ cell lymphoma of the breast, the patient underwent six courses of R-CHOP (rituximab, cyclophosphamide, hydroxydaunorubicin, oncovin, and prednisolone) chemotherapy and radiotherapy to the breast and supraclavicular lymph nodes 
Fig. 2. Ultrasonography. A large tumor (indicated by arrows) with an expansive growth pattern showed predominantly high-level internal echoes on ultrasonography.
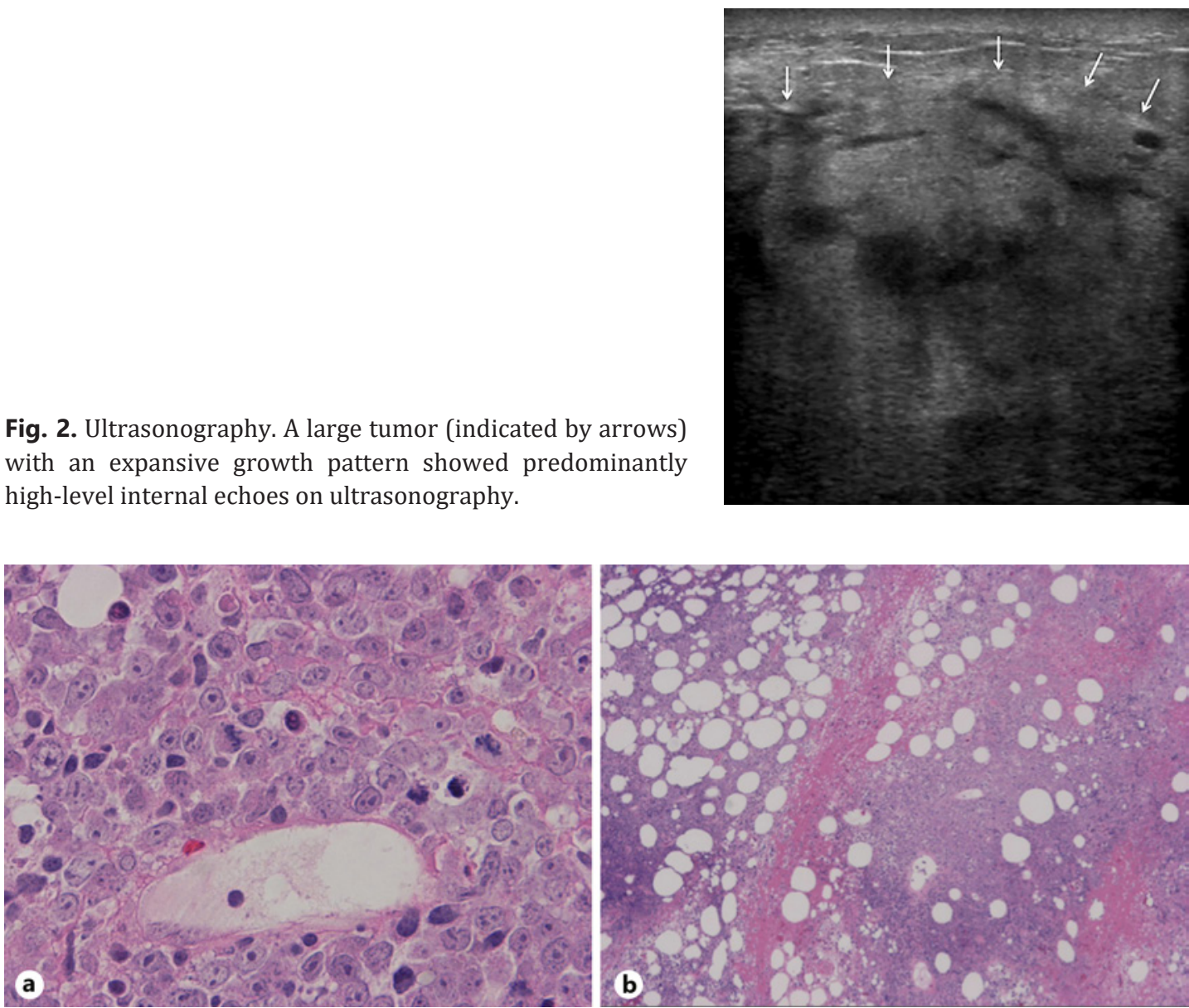

Fig. 3. Histological findings of the tumor. a The tumor cells had a large nucleus and scant cytoplasm with abundant mitoses. HE. $\times 400$. b A lot of fat cell interspersion was observed in and around the tumor cell conglomerate. HE. $\times 100$.

(maximum dose 5,270 cGy). Positron emission tomography scan taken 5 months after the completion of radiotherapy showed no tumors in the breast. The patient has been well without lymphoma recurrence for more than 6 years.

\section{Discussion}

Matching a typical image to each breast disease enables physicians to quickly master image diagnosis of breast disease, but sometimes it leads them into diagnostic pitfalls. In fact, we thought this case should be some type of malignancy due to its hypervascularity of the tumor but could not speculate what subtype of breast malignancies this case should be at the beginning of the diagnostic procedures.

To make an accurate diagnosis, in addition to the understanding of typical images of breast diseases, ultrasound diagnostic physicians should realize various factors such as wavelength, frequency, amplitude, reflection, scattering, attenuation, artifacts, focus, and acoustic impedance of the ultrasound. Of these, reflection occurs at the interface, being much larger than ultrasound wavelength, between tumor cells and normal mammary components, which 
mainly forms tumor shape. Scattering, especially posterior scattering, is a far weaker signal than reflection and shows high-level internal echoes when tumor cells commingle with nontumor cells with acoustic impedance different from that of tumor cells [7].

It is well known that impedance of fat tissue is significantly lower than that of the liver, kidney, muscle, or even blood. Therefore, interminglement with fat cells and malignant cells easily causes back scattering to make high-level internal echoes. For example, a hyperechogenic zone around breast cancer, so-called echogenic halo, appears when cancer cells invade into the fat tissue. Echogenic halo, therefore, implies that the tumor is an invasive carcinoma with invasion to the fat tissue. Thus, diagnostic physicians should pay much attention to the diagnosis of breast disease especially in patients with non-dense breast.

Malignant lymphoma of the breast has strong proliferative capacity and relatively uniform arrangement of malignant cell clusters with almost completely similar acoustic impedance, generally causing very low-level internal echoes. Lyou et al. [8] and Surov et al. [9] reported some cases of malignant breast lymphoma with partially high-level internal echoes around the central low-level internal echoes. However, they did not report a correlation between the histological findings and high-level internal echoes. We considered that lymphoma cell invasion into the fat tissue, having lower acoustic impedance than lymphoma cells [6], brought about massive back scattering and predominantly high-level internal echoes in this case. Breast composition of scattered areas of fibroglandular density should contribute to the high-level internal echoes in this case.

In conclusion, breast physicians should realize the possibility of malignant breast lymphoma with partially high-level internal echoes. Malignant breast lymphoma will never be treated with surgical intervention even when it is very small. Therefore, to further examine the correlation between pathological findings and ultrasound findings of the malignant breast lymphoma, breast physicians should harvest much more tissue using a vacuum-assisted biopsy apparatus in case of breast tumor with an expansive growth pattern and atypical ultrasound findings.

\section{Statement of Ethics}

We have reported this case in compliance with the Declaration of Helsinki. Informed consent was obtained from the patient for the publication of the clinical data.

\section{Disclosure Statement}

The authors have no conflicts of interest to declare.

\section{Funding Sources}

No funding was received for this research.

\section{Author Contributions}

All authors contributed equally to this work. 


\section{References}

1 Habbema JD, van Oortmarssen GJ, van Putten DJ, Lubbe JT, van der Maas PJ. Age-specific reduction in breast cancer mortality by screening: an analysis of the results of the Health Insurance Plan of Greater New York study. J Natl Cancer Inst. 1986;77:317-20.

2 Miller AB, To T, Baines CJ, Wall C. The Canadian National Breast Screening Study-1: breast cancer mortality after 11 to 16 years of follow-up. A randomized screening trial of mammography in women age 40 to 49 years. Ann Intern Med. 2002;137:305-12.

3 Melnikow J, Fenton JJ, Whitlock EP, Miglioretti DL, Weyrich MS, Thompson JH, et al. Supplemental screening for breast cancer in women with dense breasts: a systematic review for the U.S. Preventive services task force. Ann Intern Med. 2016;164:268-78.

4 Corsetti V, Ferrari A, Ghirardi M, Bergonzini R, Bellarosa S, Angelini O, et al. Role of ultrasonography in detecting mammographically occult breast carcinoma in women with dense breasts. Radiol Med. 2006;111:440-8.

5 The Japanese Breast Cancer Society . General rules for clinical and pathological recording of breast cancer. 18th ed. Tokyo: Kenahara-Shuppan; 2018. p. 28-9.

6 Rosen PP. Lymphoid and hematopoietic tumors. Rosen's breast pathology. 2nd ed. Philadelphia: Lippincott Williams \& Wilkins; 2001. p. 863-75.

7 Panagiotis VT. Presentation of acoustic waves propagation and their effects through human body tissue. Hum Movement. 2010;11:58-65.

8 Lyou CY, Yang SK, Choe DH, Lee BH, Kim KH. Mammographic and sonographic findings of primary breast lymphoma. Clin Imaging. 2007;31:234-8.

9 Surov A, Holzhausen H-J, Wienke A, Schmidt J, Thomssen C, Arnold D, et al. Primary and secondary breast lymphoma: prevalence, clinical signs and radiological features. Br J Radiol. 2012;85:e195-e205. 